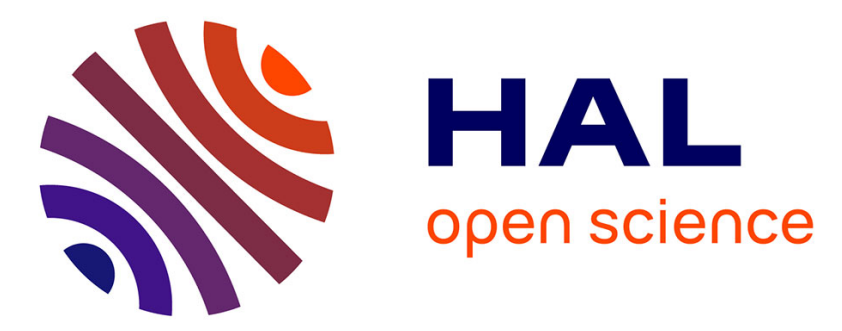

\title{
DELLA family duplication events lead to different selective constraints in angiosperms
}

Jean Keller, Pauline Delcros, Cyril Libourel, Francisco Cabello-Hurtado, Abdelkader Aïnouche

\section{- To cite this version:}

Jean Keller, Pauline Delcros, Cyril Libourel, Francisco Cabello-Hurtado, Abdelkader Aïnouche. DELLA family duplication events lead to different selective constraints in angiosperms. Genetica, 2020, 148 (5-6), pp.243-251. 10.1007/s10709-020-00102-6 . hal-02929503

\section{HAL Id: hal-02929503 https://hal.science/hal-02929503}

Submitted on 9 Nov 2020

HAL is a multi-disciplinary open access archive for the deposit and dissemination of scientific research documents, whether they are published or not. The documents may come from teaching and research institutions in France or abroad, or from public or private research centers.
L'archive ouverte pluridisciplinaire HAL, est destinée au dépôt et à la diffusion de documents scientifiques de niveau recherche, publiés ou non, émanant des établissements d'enseignement et de recherche français ou étrangers, des laboratoires publics ou privés. 


\section{DELLA family duplication events lead to different selective constraints in angiosperms.}

2 Authors: Keller J. ${ }^{1,2}$, Delcros P. ${ }^{1}$, Libourel C. ${ }^{2}$, Cabello-Hurtado F. ${ }^{1}$, Aïnouche A. ${ }^{1}$

3 Affiliation : ${ }^{1}$ UMR CNRS 6553 Ecobio, OSUR (Observatoire des Sciences de l'Univers de

4 Rennes), Université de Rennes 1, 35042 Rennes, France ; ${ }^{2}$ LRSV, Université de Toulouse,

5 CNRS, UPS, Castanet-Tolosan, France

6 Corresponding author: abdelkader.ainouche@univ-rennes1.fr

\section{Acknowledgement}

9 We acknowledge the GenOuest bioinformatics core facility (https://www.genouest.org) for providing the 10 computing infrastructure. Part of this work was conducted at the LRSV laboratory, which belongs to the 11 TULIP Laboratoire d'Excellence (LABEX) (ANR-10-LABX-41). JK and CL were supported by the research 12 project Engineering Nitrogen Symbiosis for Africa (ENSA), which is funded through a grant to the University 13 of Cambridge by the Bill \& Melinda Gates Foundation (OPP1172165). JK was supported by a doctoral 14 research grant from the University of Rennes 1 - French Ministry of Higher Education and Research. This work benefited from the International Associated Laboratory "Ecological Genomics of Polyploidy" 16 supported by CNRS (INEE, UMR CNRS 6553 Ecobio), University of Rennes 1, Iowa State University 17 (Ames, USA). 
Abstract

Gibberellic acid (GA) is a major plant hormone involved in several biological processes from the flowering to the symbiosis with microorganisms. Thus, the GA regulation is crucial for plant biology. This regulation occurs via the DELLA proteins that belong to the GRAS transcription factor family. DELLA proteins are characterised by a DELLA N-terminal and a GRAS C-terminal domains. It is well known that DELLA activity appears after the bryophytes divergence and then evolved in the vascular plant lineages. Here we present the phylogeny of DELLA across 75 species belonging to various lineages from algae, liverworts and angiosperms. Our study confirmed two main duplication events, the first occurring before the angiosperms divergence and the other specific to the eudicots lineage. Comparative analysis of DELLA subclades in angiosperms revealed the loss in Poaceae and strong alteration in other species of the DELLA functional domain in the DELLA2 clade. In addition, molecular evolution analysis suggests that each of the clades (named DELLA1.1, DELLA1.2 and DELLA2) evolved differently but copies of each subclade are under strong purifying selection. This also suggests that, although the DELLA functional domain is altered in DELLA2, DELLA2 orthologs are still functional and operate in a different way compared to DELLA1 copies. In angiosperms, additional duplication events occurred and led to duplicate copies in species, genus or family such as in the Fabaceae subfamily Papilionoideae. This duplication led to the formation of additional paralogs in the DELLA1.2 subclade (DELLA1.2.1 and DELLA1.2.2). Interestingly, both copies appeared to be under relaxing selection revealing different evolutionary fate of the DELLA duplicated copies. 


\section{Introduction}

Plant hormones play essential roles in various plant biology aspects and a huge diversity of these compounds have been identified so far (Davies 2010). Among them, the gibberellic acid (GA), a tetracyclic di-terpenoid molecule, is particularly involved in plant growth, seed germination and development or flowering (Gupta and Chakrabarty 2013). It also plays a role in responses to environmental stimuli (water, light, temperature) as well as during the symbiosis with microorganisms such as nitrogen-fixing bacteria and arbuscular mycorrhizal fungi (Lievens et al. 2005; Davies 2010; Ferguson et al. 2011; Gupta and Chakrabarty 2013; Takeda et al. 2015; Martín-Rodríguez et al. 2016)

Regulation of GA acitvity occurs through the DELLA (named according the conserved DELLA amino acid motif at the protein N-terminal domain) proteins which form a complex with the GA receptor GID1 repressing the expression of downstream GA-responsive genes (Ueguchi-Tanaka et al. 2007; Sun 2011). Fixation of GA to the GID1 receptor induces the proteasome-dependant degradation of DELLA proteins (Fu et al. 2002). This GA-GID1-DELLA complex has been observed in all the investigated vascular plants but is absent from bryophytes although a DELLA copy has been identified in Physcomitrella patens (Yasumura et al. 2007; Sun 2011). This suggests that the DELLA repressor activity of GA evolved after the bryophyte divergence ( 430 MYA) and there is no functional data about the DELLA role and mode of action prior this divergence (Kenrick and Crane 1997; Yasumura et al. 2007; Wang and Deng 2014). In angiosperms, DELLA proteins are known to be involved in the regulation of a wide range of fundamental biological processes such as flowering, root and shoot development, and is a key regulator of both arbuscular mycorrhizal and root nodule nitrogen-fixing symbiosis (Maekawa et al. 2009; Ferguson et al. 2011; Floss et al. 2013; Yu et al. 2014; Pimprikar et al. 2016). For example, during nitrogen-fixing symbiosis, DELLA is thought to be a key regulator of the infection process by directly regulating symbiotic genes NSP1/NSP2, ERN1,NF-YA1 and by enhancing the binding between CYCLOPS and NSP2 (Fonouni-Farde et al. 2016; Jin et al. 2016). Recently, DELLA proteins were also shown to be likely involved in the cytokinin genetic pathway regulation during nodulation in Pisum sativum L. (Dolgikh et al. 2019).

DELLA constitutes a subfamily of the GRAS transcription factors superfamily and DELLA proteins are characterised by a N- and C- terminal conserved DELLA and GRAS domains respectively (Pysh et al. 1999). The GRAS family encompasses between 8 and 13 subfamilies according to authors and surveyed species (Cenci and Rouard 2017). Although evolutionary history of the GRAS family has been recently investigated in representative angiosperms model species (Cenci and Rouard 2017), there is little data about the DELLAs evolution in angiosperms.

In this context, we performed a large screening of DELLA proteins in more than 50 species representing all the main plant orders. This allowed us to confirm different duplication events in the DELLA subfamily, with a subclade exhibiting severe alterations of the DELLA functional domain. In addition, molecular evolution analysis revealed that the angiosperms DELLA subclades have experienced different selective constraints with currently a strong purifying constraint within each of these subclades. 
Proteomes of 75 plant species covering the main Angiosperms lineages (list available in Supplementary Table S1) were screened against the Pfam database (Finn et al. 2016) and hits with an e-value lower than 0.01 and both GRAS (PF12041.8) and DELLA (PF03514.14) domains were retained. To complement this search and take into account potential missing domains (in cases of losses or miss-annotated proteins), published and well characterized DELLA protein from Arabidopsis thaliana, Oryza sativa, Lupinus angustifolius, Medicago truncatula and Amborella trichopoda (Sato et al. 2014; Cenci and Rouard 2017; Hane et al. 2017) were used as queries for a tBLASTn v2.7.1+ search against the 75 plant species with default parameters (Camacho et al. 2009) and an e-value threshold of $1^{-60}$. Finally, protein sequences shorter than 200 amino acids were removed from the dataset to eliminate partial assembly and putative remnants (retained and removed sequences are available in Supplementary Data S1 and S2 respectively).

In order to explore the absence of DELLA proteins in algae, the Algae-PrAS database containing 31 genomes (http://alga-pras.riken.jp/) was also screened with the reference DELLA sequences from A. thaliana and $O$. sativa as queries using the BLASTp v2.7.1+ algorithm and an e-value threshold of 0.01. Until recently, scarce data were available for Zygnematophyceae algae, the likely closest lineage of land plants. Two Zygnematophyceae genomes (Mesotaenium endlicheranium and Spirogloea muscicola, this latter belonging to a new subclass likely to be the closest from the Zygnematophyceae/embryophytes separation) have been published and were screened for DELLA homologs (Cheng et al. 2019).

\section{Phylogenetic analysis}

99 Retained protein sequences were aligned using MUSCLE (Edgar 2004) with default parameters. Alignment 100 was subjected to maximum likelihood analysis using IQ-TREE v1.5.5 (Nguyen et al. 2015). The best-fitted evolutionary model was determined using ModelFinder as implemented in IQ-TREE (Kalyaanamoorthy et al. 2017). Maximum likelihood analysis was conducted with 10,000 replicates Ultra Fast Bootstraps (Hoang et al. 2018). Trees were visualized and annotated uing the Interactive Tree of Life platform (Letunic and Bork 2016).

\section{Molecular evolution analyses}

106 For each clade of interest identified through the phylogenetic analysis, sequence logos were generated from 107 protein alignments using the WebLogo v3.5 platform with default parameters (Crooks 2004). In addition, to 108 compare signature of selection among DELLA homolog clades identified in phylogeny, we estimated dN/dS ratios from all pairwise comparison of protein-coding sequences using yn00 module from the PAML package $110 \quad v 4.9 \mathrm{~g}$ (Yang and Nielsen 2000; Yang 2007). We also used the RELAX hypothesis testing framework from 111 HyPhy (Pond et al. 2005; Wertheim et al. 2015) to look for relaxation $(K<1)$ or intensification $(K>1)$ of the 112 signature of selection. To estimate signature of selection, codons were aligned according to the protein 113 alignment using the pal2nal v14 Perl script (Suyama et al. 2006) and all gapped positions were removed. We 
114 used the following command line for each clade tested (SupplementaryTable2): hyphy relax CPU=1 --

115 alignment input_file.fna --code Universal --type Nucleotide --keyword value Foreground.

\section{Results and discussion}

118 DELLA family emerged with land plants and experienced multiple duplication events

119 To understand the origin of the DELLA family, the Algae-PrAS database that contains more than 30 genomes 120 of non-land plants species (green and red algae, Glaucophyceae) and other organisms such as Oomycetes and 121 diatoms was mined using DELLA protein sequences from Arabidopsis thaliana and Oryza sativa. Results 122 confirmed the absence of DELLA orthologs in these species. In the two Zygnematophyceae genomes, no 123 DELLA were detected but other GRAS family members were identified and are likely resulting from 124 horizontal gene transfer from bacteria (Cheng et al. 2019). These findings are consistent with results from 125 Yasamura et al. (2007) who suggested an emergence of DELLA proteins along with the land colonization by 126 plants. To improve our understanding of the evolutionary dynamics of DELLA family, 75 land plants 127 genomes were investigated and 293 DELLA orthologs were identified by the phylogenetic analysis 128 (excluding putative remnants and misassembled sequences). Among them, one sequence with a DELLA 129 functional domain was identified in the liverwort Marchantia polymorpha (Marpol_Mapoly0058s0044.1) 130 consistently with the hypothesis that the DELLA family originated at least in the common ancestor of the 131 vascular and non-vascular plants (Hernández-García et al. 2019). Previous findings revealed that the GID1132 DELLA complex does not exist in the bryophytes lineage (Yasumura et al. 2007; Hirano et al. 2007). In 133 bryophytes, DELLA has been recently proposed as transcriptional co-activator that could have been exapted 134 in vascular plants (Hernández-García et al. 2019). Previously published results based on RNAseq data and 135 putative DELLA's interactors in Physcomitrella patens (no DELLA-GID1 interaction), Solanum 136 lycopersicum and Arabidopsis thaliana suggest that DELLA evolution is correlated with an increasing 137 coordination of transcriptional programs between bryophytes and vascular plants (Briones-Moreno et al. 138 2017).

139 Phylogenetic analysis shows that DELLA family underwent multiple duplication events at different points 140 of the land plant evolution as recently shown by Hernández-Garcia and colleagues (2019). Indeed, results 141 revealed a first duplication event that occurred before the divergence of angiosperms (Fig.1, with branch 142 support values to be retrieved in the Supplementary Data S3) leading to the formation of two main clades, 143 called DELLA2 (blue clade in Fig.1; corresponding to DELLA3 in Hernández-Garcia et al. 2019) and 144 DELLA1 (orange, green and red clades) accordingly to Cenci and Rouard (2017). DELLA sequences from 145 Marchantia polymorpha and the lycophyte Selaginella moellendorffii seem to belong to the DELLA2 clade; 146 however, these branches are not supported (UFB value $<50 \%$ ) and these sequences are likely at the base of 147 the whole DELLA family. As demonstrated in recent phylogeny and in this study the DELLA1/DELLA2 148 clades emergence is probably due to the Whole Genome Duplication (WGD) event that occurred prior the 149 seed plants (c.a 319 MYA) (Jiao et al. 2011; Hernández-García et al. 2019). Another level of duplication has 
been identified in the DELLA1 clade. Indeed, this latter, with the basal Angiosperms Amborella trichopoda

151 at its base, can be divided into three main subclades (Fig.1). The first, in green, is exclusively composed by

152 Monocot species whereas the two others only contain Eudicot species. These two Eudicot subclades emerged

153 from the core Eudicot WGD event that occurred approximately 125 MYA (Van de Peer et al. 2017) and were

154 arbitrarily named DELLA1.1 (orange clade in Fig.1) and DELLA1.2 (red clade). Finally, additional

155 duplication events limited to species or subclades were identified such as in Brassicaceaee DELLA1.1 or the

156 DELLA1.2 of Papilionoideae (WGD of the Papilionoideae common ancestor, around 60 MYA, see below).

157 For each sequence, gene structure was extrapolated from the annotation file, when available, and results were

158 mapped on the phylogeny (Fig. 1; outer circle). Excepting few sequences, most of the genes present a non-

159 intronic structure and a length ranked between 1000 and $2000 \mathrm{bp}$.

160 Analysis of the functional domains revealed alterations in DELLA2

161 DELLA proteins are characterised by the presence of two functional domains. The first (DELLA) is in the

162 N-terminal region of the protein and is specific to the DELLA family whereas the second (GRAS) is shared

163 with other members of the GRAS superfamily. Functional analysis of the DELLA domain in the model plant

164 Arabidopsis thaliana characterised two motifs required for the interaction with GID1 (Murase et al. 2008):

165 DELLA§LXYXVXXXXMAXVAXXLEXLEX§ and TVHYNPXXLXXWXXXM (where X and § stand for 166 any and non-polar amino acids respectively).

167 DELLA and GRAS functional domains identified using the Pfam search were mapped on the phylogenetic 168 tree (Fig.1, second outer circle) and revealed that most of the sequences possess both domains with few 169 exceptions that can be explained by assembly and annotation issues or putative pseudogenes. However, it 170 appears that all the six Poaceae from the DELLA2 clade have lost the DELLA functional domain (absence 171 of red right pointing pentagram in Fig.1, and Supplementary Figure 1). The loss of the DELLA domain has 172 been previously reported in rice (Itoh et al. 2005; Cenci and Rouard 2017) and our results indicate that this 173 loss is likely to have occurred before diversification of the Poaceae from their common ancestor. Indeed, 174 Monocots from other orders such as Spirodela polyrhiza, Zostera marina (two Alismatales) and Musa 175 accuminata (Zingiberales) retained both DELLA and GRAS domains (Supplementary Figure 1). At the 176 opposite, all Monocots DELLA1 proteins have a full DELLA domain. Previous functional analysis of the 177 two DELLA2 copies of rice (also called SLRL1 and SLRL2 for SLR-like1 and 2) revealed that SLRL1 is 178 positively affected by GA treatment whereas SLRL2 is not. In addition, trans-complementation of slr1 179 (DELLA1) mutant by SLRL1 rescued the phenotype (Itoh et al. 2005). Since the N-terminal DELLA domain 180 is missing in SLRL1 and 2, proteins were not degraded following the GA treatment but also exhibit a lower 181 activity against GA than a SLR1 truncated copy missing the DELLA domain (Itoh et al. 2005). Itoh and 182 colleagues suggest that SLR1 and SLRL1 can function together and are required for a fine-tuning of GA 183 signalling.

184 In a second approach, the focus has been made on the DELLA functional domain extracted from the protein 185 alignment and amino acids conservation represented using the WebLogo tool (Crooks 2004) for each main 
clade of DELLA (Fig.2). Results revealed a high conservation of amino acids required for interaction with GID1 across the different DELLA1 subclades (DELLA1.1, DELLA1.2 and DELLA1 from Monocots). However, severe disruption of the DELLA motifs can be observed in the DELLA2 clade (Fig.2; red arrows). Indeed, the methionine from the MAXVA motif and at the end of the second DELLA subdomains is not conserved anymore among the DELLA2 sequences. Other disruptions of the DELLA domain are also characteristic of the DELLA2 clade such as the low conservation of the first leucine in the DELLA first motif or the alteration of the TV motif in the second DELLA motif (Fig.2). This low-conservation pattern observed in the DELLA2 clade could be explained by different hypothesis such as the pseudogenisation of the DELLA2 copies, a new function of these proteins, or another way for these proteins to interact with GID1.

To test whether the DELLA2 clade is under relaxed selective constraints, different evolutionary tests were applied. To this aim, only sequences with entire DELLA domains were retained and evolutionary tests performed on the domain alignment. The analysis of non-synonymous versus synonymous (dN/dS) revealed a higher dN/dS ratio when comparing DELLA2 to the other DELLA1 subclades, whereas these latter had similar dN/dS ratios (Fig.3). To determine if relaxed selection is acting on the DELLA2 clade, a branch model (RELAX) was applied with the DELLA2 clade marked as foreground branch (Supplementary Table 2). According to pairwise comparisons, estimating the selective intensity parameter $(\mathrm{K})$ suggests that the DELLA2 clade is subjected to an ongoing process of relaxing selection $(K=0.81$, $p$-val $\approx 0.00074, L R=11.39$ ). Conversely, the higher value found for DELLA1 $(\mathrm{K}=1.28$, $\mathrm{p}$-val $\approx 0.00038, \mathrm{LR}=12.61)$ suggests intensification of the purifying selection $(K>1)$ to maintain the proper role of DELLA1. In addition, logo and amino acid frequency for each position of the DELLA motifs (Fig.2 and Supplementary Table S3) show that mutations of essential amino acid in DELLA2 clade are shared by most of the sequences in this clade. For example, methionine from MAXVA stretch is replaced by leucine in $100 \%$ of sequences. Similarly, almost $90 \%$ of DELLA2 sequences share a glycine instead of a glutamate normally expected at the second position of the DELLA first motif. Altogether, these analyses suggest that modification of DELLA2 sequences occurred in the DELLA2 ancestor and then, have been maintained. These results are in line with a previous report suggesting that positive selection acted on the DELLA family during its evolution (Chen et al. 2013). To date, there is no detailed studies of DELLA2 proteins from Eudicot lineage. However, it is possible that, similarly to Poaceae DELLA2, these proteins function along with their DELLA1 counterparts and act as weaker GA signalling repressors without being degraded. Interestingly, it has been shown that the RGL1 protein of Arabidopsis thaliana (DELLA1.2 subclade) works without being degraded upon GA application (Wen and Chang 2002). All together, these results suggest that the multiple rounds of duplication in the DELLA family led to a slight subfunctionalization of DELLA copies, all involved in the GA regulation pathway. In their work, Yasumura and colleagues (2007), proposed a stepwise evolution of the DELLA-GA complex in plants with the GID1-DELLA interaction evolving in vascular plants. Here, we show that the DELLA family pursued its evolution in Angiosperms by experiencing several rounds of duplication, leading to sub-clades under different selective constraint. In addition, our results revealed that the loss of DELLA domain is shared among the DELLA2 proteins of Poaceae, supporting the hypothesis of a different mechanism (degradation-independent for example) for DELLA in this family (Itoh et al. 2005). 
225 DELLA proteins play major role in various biological aspects of plant life. In legumes, they also have a 226 critical role for the establishment of the root nodule nitrogen-fixing symbiosis. Recently, DELLA proteins 227 have been proposed to be also involved in the regulation of cytokinin genetic pathway through interaction 228 with KNOX and BELL transcription factors (Dolgikh et al. 2019). Thus, a focused analysis on the DELLA 229 sequences from the Fabaceae family has been performed and showed similar evolutionary pattern compared 230 to results discussed above.

231 First, the evolutionary pattern of DELLAs confirmed that the three main DELLA clades (DELLA2, 232 DELLA1.1 and DELLA1.2) are conserved among the Fabaceae (Fig.4A). DELLA1 and DELLA2 copies 233 both present mutations in amino acids involved in the interaction with GID1 (Fig.4B). The branch model 234 analysis on both clades showed that DELLA1 and DELLA2 copies have a similar pattern of selection with 235 no sign of relaxation/intensification (Supplementary Table S2). However, no change in the selective 236 constraint was detected among the different clades suggesting that, also in Papilionoideae, there is no positive 237 selection acting on them.

238 Second, in the DELLA1.2 subclade, an additional duplication event occurred leading to the formation of two 239 subclades arbitrarily called DELLA1.2.1 and DELLA1.2.2. These two subclades likely originated from the 240 Papilionoideae WGD that occurred 60MYA. Analysis of the selective constraints strongly suggests that both 241 paralogs $(\mathrm{K}=0.25$, $p$-val=7.3E-08, $\mathrm{LR}=28.98$ and $\mathrm{K}=0.26$, $p$-val=7.2E-08, LR=29 for DELLA1.2.1 and 242 DELLA1.2.2 respectively) are under pseudogenization process. This is also supported by the apparent loss of DELLA1.2.1 copy in seven out of the 15 legume samples investigated.

\section{Conclusion}

246 DELLA proteins have a critical role in numerous aspects of plant life, from development to flowering but also in specific aspects such as in the nitrogen-fixing symbiosis. Phylogenetic analysis of DELLA family including more than 70 species confirmed its emergence in land plants along with its absence in algae. Results of phylogenetic analysis of the DELLA family is consistent with previous reports and show the existence of two main duplication events leading to the formation of three clades: DELLA1 and DELLA2 clades emerged from a duplication event that occurred before the Angiosperms divergence and two additional DELLA1 subclades (DELLA1.1 and DELLA1.2) emerged following the Eudicots whole genome duplication. A detailed analysis of the evolution of the three DELLA clades revealed that they are all under purifying selection although their ancestors seem to have been subjected to a relaxed selective constraint. In the DELLA2 clade, our results strongly support the loss of the DELLA domain before diversification of Poaceae and severe alterations of this motif in other DELLA2 proteins suggesting that DELLA2 protein could function in a degradation-independent manner. Finally, DELLA proteins from the Fabaceae family revealed a similar 
ancestor leading to additional DELLA1.2.1 and DELLA1.2.2 clades. Both clades are under relaxing selection suggesting they are likely to be pseudogenized or neofunctionalized.

All together this study supports the hypothesis of a complex evolutionary history of DELLA proteins in Angiosperms with multiple changes in the selective constraint. To extend these results, functional data on the different DELLA copies from both Monocots and Eudicots would be of a great interest to better understand the biological consequences of these differential selective constraints.

\section{Conflict of interest}

The authors declare that they have no conflict of interest.

\section{References}

Briones-Moreno A, Hernández-García J, Vargas-Chávez C, et al (2017) Evolutionary Analysis of DELLA-Associated Transcriptional Networks. Front Plant Sci 8:626. https://doi.org/10.3389/fpls.2017.00626

Camacho C, Coulouris G, Avagyan V, et al (2009) BLAST+: architecture and applications. BMC Bioinformatics 10:421. https://doi.org/10.1186/1471-2105-10-421

Cenci A, Rouard M (2017) Evolutionary Analyses of GRAS Transcription Factors in Angiosperms. Front Plant Sci 8:. https://doi.org/10.3389/fpls.2017.00273

Chen J, Cheng T, Wang P, et al (2013) Genome-wide bioinformatics analysis of DELLA-family proteins from plants. Plant Omics Journal, POJ 6(3):201-207

Cheng S, Xian W, Fu Y, et al (2019) Genomes of Subaerial Zygnematophyceae Provide Insights into Land Plant Evolution. Cell 179:1057-1067.e14. https://doi.org/10.1016/j.cell.2019.10.019

Crooks GE (2004) WebLogo: A Sequence Logo Generator. Genome Res 14:1188-1190. https://doi.org/10.1101/gr.849004

Davies PJ (ed) (2010) Plant Hormones. Springer Netherlands, Dordrecht

Dolgikh AV, Kirienko AN, Tikhonovich IA, et al (2019) The DELLA Proteins Influence the Expression of Cytokinin Biosynthesis and Response Genes During Nodulation. Front Plant Sci 10: 432. https://doi.org/10.3389/fpls.2019.00432

Edgar RC (2004) MUSCLE: multiple sequence alignment with high accuracy and high throughput. Nucleic Acids Res 32:1792-1797. https://doi.org/10.1093/nar/gkh340

Ferguson BJ, Foo E, Ross JJ, Reid JB (2011) Relationship between gibberellin, ethylene and nodulation in Pisum sativum. New Phytol 189:829-842. https://doi.org/10.1111/j.14698137.2010.03542.x 
Finn RD, Coggill P, Eberhardt RY, et al (2016) The Pfam protein families database: towards a more sustainable future. Nucleic Acids Res 44:D279-D285. https://doi.org/10.1093/nar/gkv1344

Floss DS, Levy JG, Levesque-Tremblay V, et al (2013) DELLA proteins regulate arbuscule formation in arbuscular mycorrhizal symbiosis. Proc Natl Acad Sci 110:E5025-E5034. https://doi.org/10.1073/pnas.1308973110

Fonouni-Farde C, Tan S, Baudin M, et al (2016) DELLA-mediated gibberellin signalling regulates Nod factor signalling and rhizobial infection. Nat Commun 7:. https://doi.org/10.1038/ncomms12636

Fu X, Richards DE, Ait-ali T, et al (2002) Gibberellin-Mediated Proteasome-Dependent Degradation of the Barley DELLA Protein SLN1 Repressor. Plant Cell 14:3191-3200. https://doi.org/10.1105/tpc.006197

Gupta R, Chakrabarty SK (2013) Gibberellic acid in plant: Still a mystery unresolved. Plant Signal Behav 8:e25504. https://doi.org/10.4161/psb.25504

Hane JK, Ming Y, Kamphuis LG, et al (2017) A comprehensive draft genome sequence for lupin (Lupinus angustifolius ), an emerging health food: insights into plant-microbe interactions and legume evolution. Plant Biotechnol J 15:318-330. https://doi.org/10.1111/pbi.12615

Hernández-García J, Briones-Moreno A, Dumas R, Blázquez MA (2019) Origin of GibberellinDependent Transcriptional Regulation by Molecular Exploitation of a Transactivation Domain in DELLA Proteins. Mol Biol Evol 36:908-918. https://doi.org/10.1093/molbev/msz009

Hirano K, Nakajima M, Asano K, et al (2007) The GID1-Mediated Gibberellin Perception Mechanism Is Conserved in the Lycophyte Selaginella moellendorffii but Not in the Bryophyte Physcomitrella patens. Plant Cell 19:3058-3079. https://doi.org/10.1105/tpc.107.051524

Hoang DT, Chernomor O, von Haeseler A, et al (2018) UFBoot2: Improving the Ultrafast Bootstrap Approximation. Mol Biol Evol 35:518-522. https://doi.org/10.1093/molbev/msx281

Itoh $\mathrm{H}$, Shimada A, Ueguchi-Tanaka M, et al (2005) Overexpression of a GRAS protein lacking the DELLA domain confers altered gibberellin responses in rice: Rice SLR1-like genes. Plant J 44:669-679. https://doi.org/10.1111/j.1365-313X.2005.02562.x

Jiao Y, Wickett NJ, Saravanaraj A, et al (2011) Data from: Ancestral polyploidy in seed plants and angiosperms. Nature 473 (7345): 97 100. https://doi.org/10.1038/nature09916.

Jin Y, Liu H, Luo D, et al (2016) DELLA proteins are common components of symbiotic rhizobial and mycorrhizal signalling pathways. Nat Commun 7:(1). https://doi.org/10.1038/ncomms 12433 
Kalyaanamoorthy S, Minh BQ, Wong TKF, et al (2017) ModelFinder: fast model selection for accurate phylogenetic estimates. Nat Methods 14:587-589. https://doi.org/10.1038/nmeth.4285

Kenrick P, Crane PR (1997) The origin and early evolution of plants on land. Nature 389 (6646): 33 39Nature 389:33-39. https://doi.org/10.1038/37918

Letunic I, Bork P (2016) Interactive tree of life (iTOL) v3: an online tool for the display and annotation of phylogenetic and other trees. Nucleic Acids Res 44:W242-W245. https://doi.org/10.1093/nar/gkw290

Lievens S, Goormachtig S, Den Herder J, et al (2005) Gibberellins Are Involved in Nodulation of Sesbania rostrata. Plant Physiol 139:1366-1379. https://doi.org/10.1104/pp.105.066944

Maekawa T, Maekawa-Yoshikawa M, Takeda N, et al (2009) Gibberellin controls the nodulation signaling pathway in Lotus japonicus. Plant J 58:183-194. https://doi.org/10.1111/j.1365-313X.2008.03774.x

Martín-Rodríguez JA, Huertas R, Ho-Plágaro T, et al (2016) Gibberellin-Abscisic Acid Balances during Arbuscular Mycorrhiza Formation in Tomato. Front Plant Sci 7:. https://doi.org/10.3389/fpls.2016.01273

Nguyen L-T, Schmidt HA, von Haeseler A, Minh BQ (2015) IQ-TREE: A Fast and Effective Stochastic Algorithm for Estimating Maximum-Likelihood Phylogenies. Mol Biol Evol 32:268-274. https://doi.org/10.1093/molbev/msu300

Pimprikar P, Carbonnel S, Paries M, et al (2016) A CCaMK-CYCLOPS-DELLA Complex Activates Transcription of RAM1 to Regulate Arbuscule Branching. Curr Biol 26:987998. https://doi.org/10.1016/j.cub.2016.01.069

Pond SLK, Frost SDW, Muse SV (2005) HyPhy: hypothesis testing using phylogenies. Bioinformatics 21:676-679. https://doi.org/10.1093/bioinformatics/bti079

Pysh LD, Wysocka-Diller JW, Camilleri C, et al (1999) The GRAS gene family in Arabidopsis: sequence characterization and basic expression analysis of the SCARECROW-LIKE genes. Plant J 18:111-119. https://doi.org/10.1046/j.1365-313X.1999.00431.x

Sato T, Miyanoiri Y, Takeda M, et al (2014) Expression and purification of a GRAS domain of SLR1, the rice DELLA protein. Protein Expr Purif 95:248-258. https://doi.org/10.1016/j.pep.2014.01.006

Sun T (2011) The Molecular Mechanism and Evolution of the GA-GID1-DELLA Signaling Module in Plants. Curr Biol 21:R338-R345. https://doi.org/10.1016/j.cub.2011.02.036

Suyama M, Torrents D, Bork P (2006) PAL2NAL: robust conversion of protein sequence alignments into the corresponding codon alignments. Nucleic Acids Res 34:W609-

Takeda N, Handa Y, Tsuzuki S, et al (2015) Gibberellin regulates infection and colonization of host roots by arbuscular mycorrhizal fungi. Plant Signal Behav 10:e1028706. https://doi.org/10.1080/15592324.2015.1028706 
Ueguchi-Tanaka M, Nakajima M, Motoyuki A, Matsuoka M (2007) Gibberellin Receptor and Its Role in Gibberellin Signaling in Plants. Annu Rev Plant Biol 58:183-198. https://doi.org/10.1146/annurev.arplant.58.032806.103830

Van de Peer Y, Mizrachi E, Marchal K (2017) The evolutionary significance of polyploidy. Nat Rev Genet 18:411-424. https://doi.org/10.1038/nrg.2017.26

Wang Y, Deng D (2014) Molecular basis and evolutionary pattern of GA-GID1-DELLA regulatory module. Mol Genet Genomics 289:1-9. https://doi.org/10.1007/s00438-0130797-x

Wen C-K, Chang C (2002) Arabidopsis RGL1 Encodes a Negative Regulator of Gibberellin Responses. Plant Cell 14:87-100. https://doi.org/10.1105/tpc.010325

Wertheim JO, Murrell B, Smith MD, et al (2015) RELAX: Detecting Relaxed Selection in a Phylogenetic Framework. Mol Biol Evol 32:820-832. https://doi.org/10.1093/molbev/msu400

Yang Z (2007) PAML 4: Phylogenetic Analysis by Maximum Likelihood. Mol Biol Evol 24:1586-1591. https://doi.org/10.1093/molbev/msm088

Yang Z, Nielsen R (2000) Estimating Synonymous and Nonsynonymous Substitution Rates Under Realistic Evolutionary Models. Mol Biol Evol 17:32-43. https://doi.org/10.1093/oxfordjournals.molbev.a026236

Yasumura Y, Crumpton-Taylor M, Fuentes S, Harberd NP (2007) Step-by-Step Acquisition of the Gibberellin-DELLA Growth-Regulatory Mechanism during Land-Plant Evolution. Curr Biol 17:1225-1230. https://doi.org/10.1016/j.cub.2007.06.037

Yu N, Luo D, Zhang X, et al (2014) A DELLA protein complex controls the arbuscular mycorrhizal symbiosis in plants. Cell Res 24:130-133. https://doi.org/10.1038/cr.2013.167

\section{Figure legends}

Fig. 1: Maximum likelihood tree of DELLA proteins (model JTT+F+R10). Tree was rooted using three SCARECROW (SCR, belonging to another subfamily of GRAS transcription factor) sequences from liverworts Marchantia polymorpha and are collapsed in the "outgroup" clade. DELLA2, Monocots DELLA1, Eudicots DELLA1.1 and DELLA1.2 are indicated by coloured branches as follow: blue, green, orange, red, respectively. The first outer circle indicates the main Angiosperm lineages as described below the tree. The second outer circle represents the presence/absence and the position of DELLA (red right pointing pentagram) and GRAS (dark blue rectangle) domains. Size and position of symbols is proportional to their length and position in the protein. The third outer circle illustrates the gene structure, extrapolated from the annotation file when available. Blue squares represent gene exons, their size and position are proportional to their length and positions in the gene. Branch support was tested using the UFB2 method and 
were masked from the tree for readability reason. Support values can be retrieved in the Supplementary Data S3 corresponding to the rooted tree shown here in Newick format.

407 Fig. 2: Logos of functional DELLA domains generated for each phylogenetic clade. For DELLA2, strongly affected sites are indicated by red arrows. Size of letter is proportional to the amino acid conservation at a given site. Blank in the logo represents highly gapped sites in the alignment. Theoretical functional models are written in black above logos and essential amino acids for the interaction with GID1, as defined by Murase et al. (2008), are indicated in bold.

412 Fig. 3: Boxplot representing pairwise dN/dS comparisons among the DELLA clades. All pairwise 413 comparison between all DELLA sequences were calculated. For each clade (X-axis), comparison with other 414 clades and itself (white waves) are shown separately. The dN/dS ratios were estimated using the Yang \& 415 Nielsen method (Yang and Nielsen 2000).

416 Fig. 4: Maximum likelihood tree of DELLA proteins (model JTT+ R5) in the Papilionoideae clade (A). 417 Alignment of DELLA functional domains mapped on the phylogenetic tree. Blue background is proportional 418 to identity level for each site. Functional DELLA subdomains are indicated by red rectangles (B).

\section{Supplementary Data}

421 Supplementary Data S1: Protein sequences used for phylogenetic analysis

422 Supplementary Data S2: Protein sequences discarded prior the phylogenetic analysis (less than 200 amino 423 acids)

424 Supplementary Data S3: Rooted tree presented in Figure 1 in Newick format.

425 Supplementary Table S1: Summary table of DELLA sequences repartition. For each species, sequence ID is 426 indicated for each DELLA clade. Absence of sequence for a given species/clade is indicated by empty cells. 427 In addition, order, family, reference and abbreviation used for each species are indicated in the last four 428 columns. For Vitis vinifera, the DELLA2 sequence is indicated but not retained in the analysis since it seems 429 that this sequence is missassembly.

430 Supplementary Table S2: Results of the branch analysis for signature of selection acting on the different 431 DELLA clades.

432 Supplementary Table S3: Amino acid frequency matrices for each DELLA copy. Numbers represent 433 percentage of each amino acid at each position in the DELLA functional domain. First column indicates the 434 consensus of DELLA domain. 
436 Supplementary Figure 1: Alignment of DELLA domains in DELLA2 Monocots and Medicago truncatula as 437 reference. Blue-coloured names represent sequences with a predicted DELLA domain by Pfam. The DELLA 438 functional domains are indicated by black squares with the theoretical sequence indicated below. Musa 439 acuminate, Siprodela polyrhiza and Zostera marina belong to diverse lineages of Monocots outside the 440 Poaceae clade. 


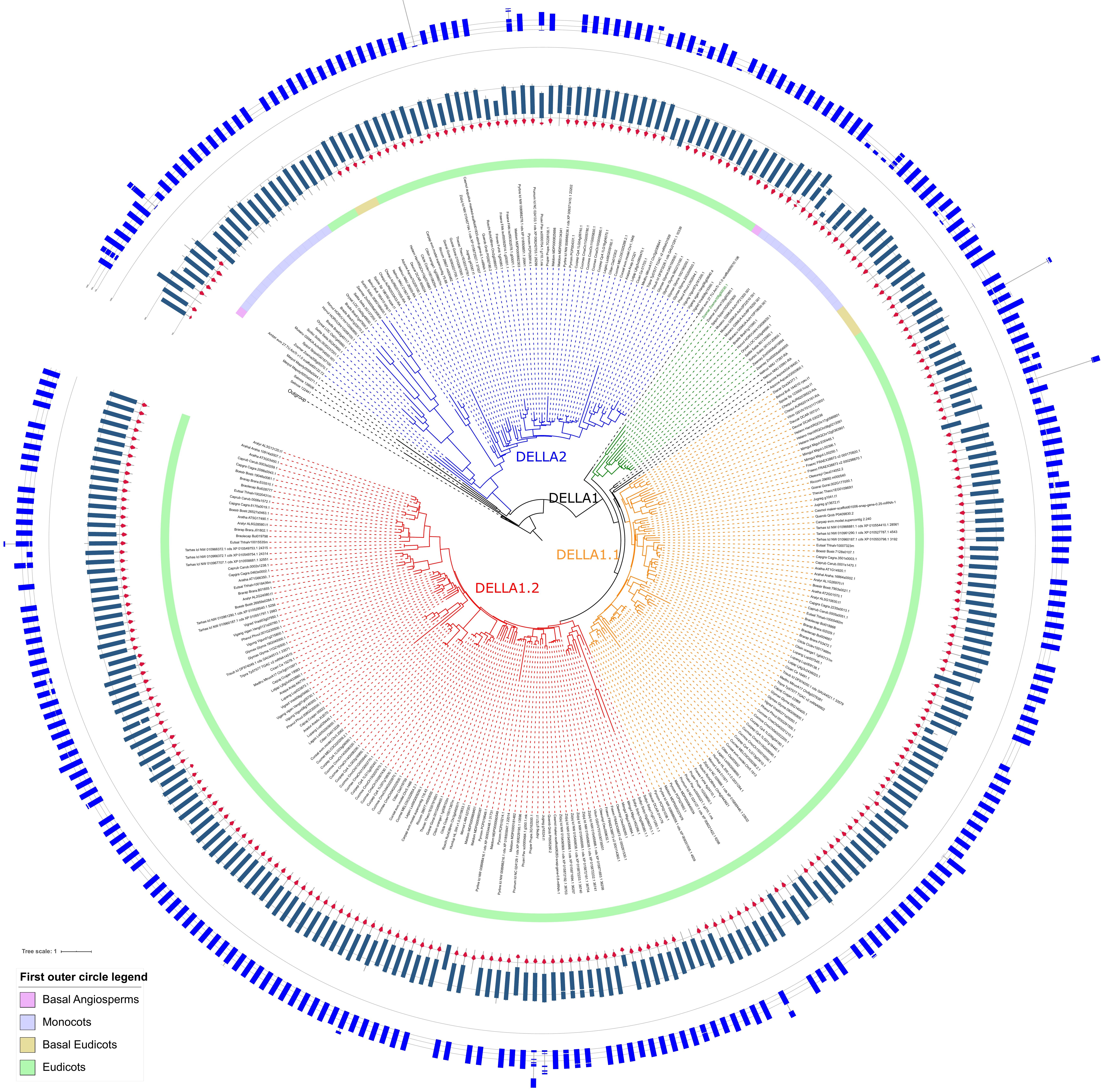


DELLA§LxYxVxxxxMAxVAxxLExLEx§

DELLA2

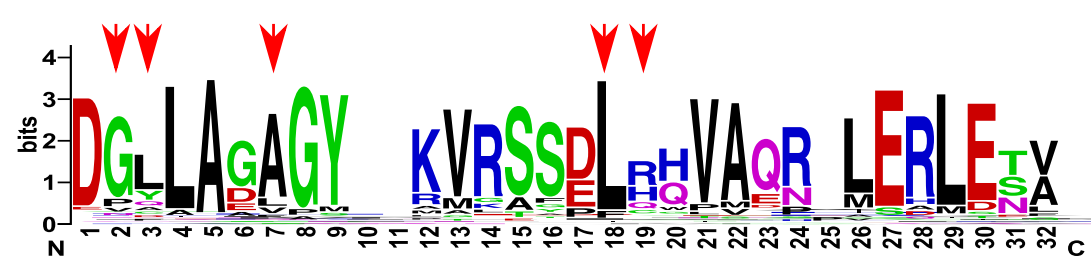

DELLA1 Monocots

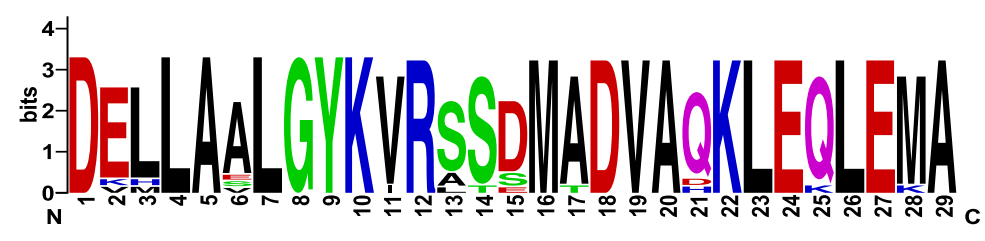

DELLA1.1 Eudicots

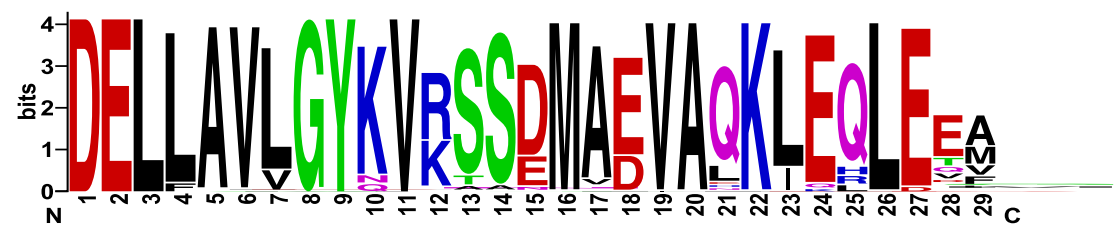

DELLA1.2 Eudicots
TVHYNPxxLxxWxxxM
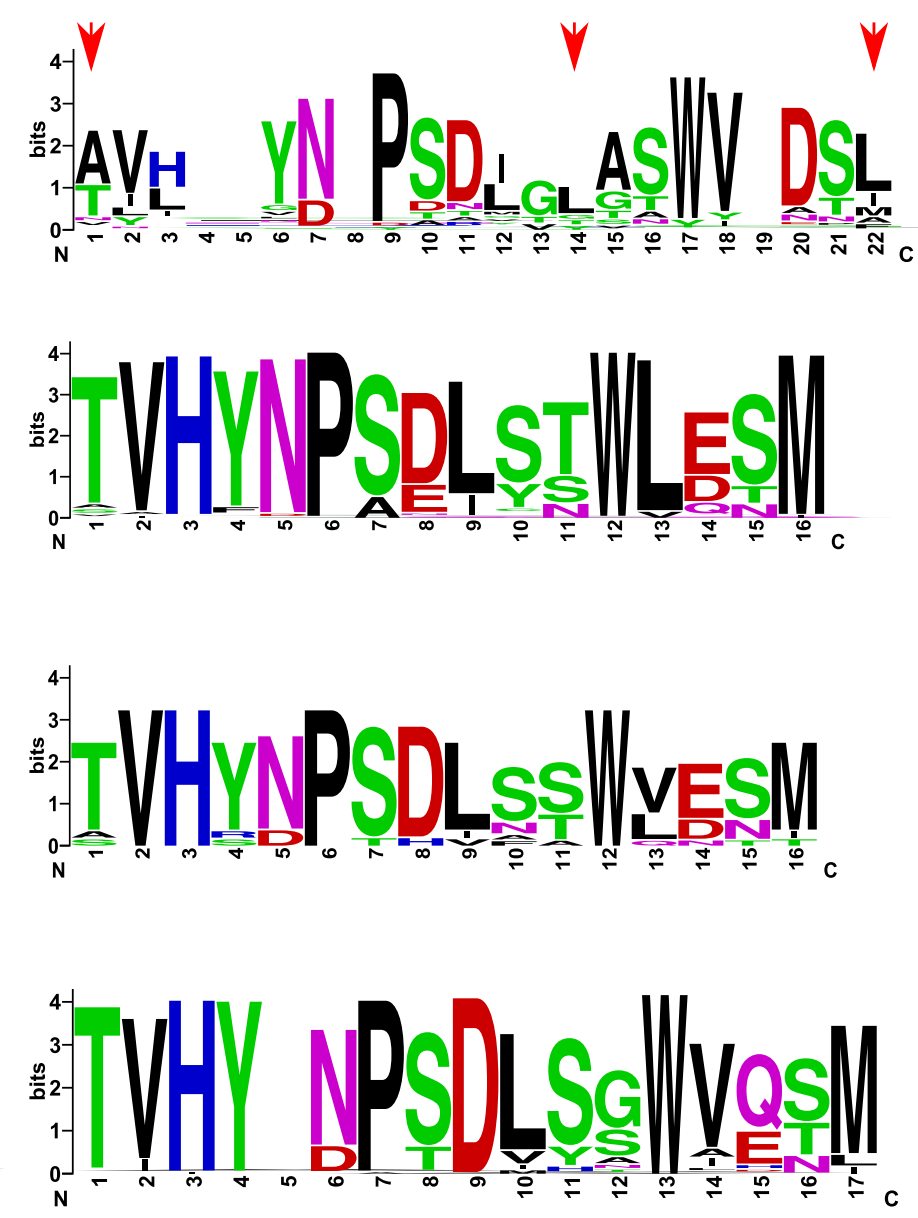

$\int_{61} \quad 97$

Vigung Vigun07g181200.1

Vigang vigan. Vang06g09640.4 Vigrad Vradi08g15350.1

64

\begin{tabular}{l}
64 \\
93 \\
\hline
\end{tabular} Araipa Araip.51Q21

Lotjap Lj6g3v0959470.1

Cicari Ca 01703.1

Medtru MtrunA17 Chr2g0306641

Tripra Tp57577 TGAC v2 mRNA31939

Trisub Icl DF973226.1 cds GAU21281.1 10339

Lupang Lup007545.1

upang Lup009138.1

Glymax Glyma.05G140400.1

Glymax Glyma.08G095800.1

Vigrad Vradi0271s00050.1

Phavul Phvul.003G291500.1

Lotjap Lj4g3v2436020.1

Cicari Ca 16491.1

Medtru MtrunA17 Chr8g0376381

Tripra Tp57577 TGAC v2 mRNA9902

Trisub Icl DF974050.1 cds GAU44521.1 33579 $95_{-}$

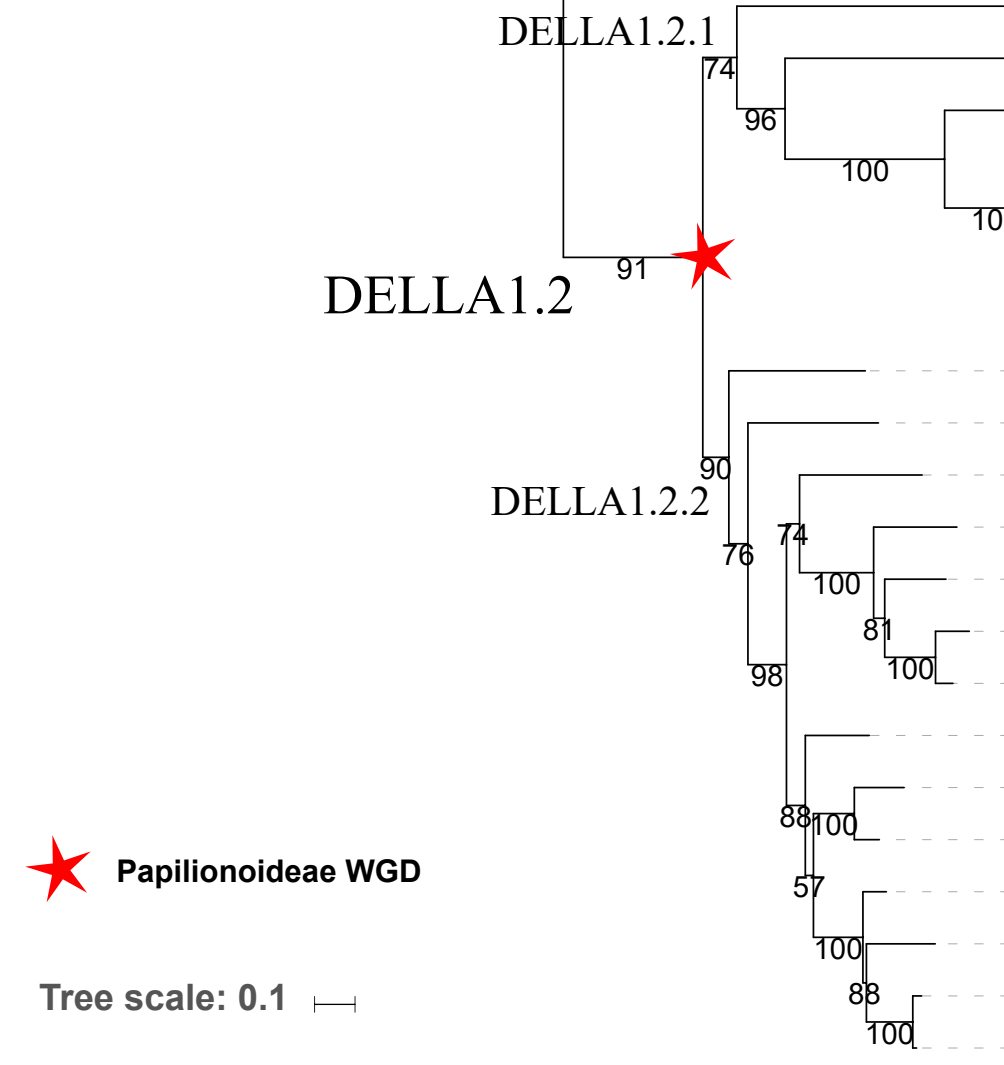

Aradur Aradu.XUD70

Cajcaj Ccajan 05522

Phavul Phvul.008G235500.

Vigung Vigun08g145500.1

Vigang vigan.Vang01g05730.

Vigrad Vradi06g05480.1

Lupang Lup023873.1

Araipa Araip.KK7TK

Lotjap Lj6g3v0433880.1

Medtru MtrunA17 Chr3g0110971

Cicari Ca 15278.1

Tripra Tp57577 TGAC v2 mRNA14515

-Trisub Icl DF974049.1 cds GAU44513.1 33571

Cajcaj Ccajan 19583

Glymax Glyma.11G216500.1

Glymax Glyma.18G040000.1

Phavul Phvul.001G230500.1

Vigung Vigun01g215800.1

Vigang vigan.Vang0121s00760.1

Vigrad Vradi03g07950.1
DGQLAGLGY - KVRSSE - LCOVAENME - RLENA INTVNSSRD - . . . ISOVVS - DALLY - DPSN IGLGSWVTL GQL AGLGY - KVRSSE - LCQVAENME - RLENA INTVNSSRD - . . . ISOVVS - DALLY - DPSN IGLGSWVDTL

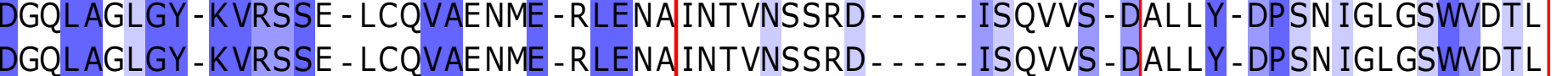
DGQL AGLGY - KVRSSE - LCQVAENME - RLENA INTVNSSRD - - - - ISQVVS - DALLY - DPSN IGLGSWVDTL DGQLAGLGY -KVRSSE - LCQVAENME - RLENA INTVNSSRD - - - - ISQVVS - DALLY - DP SN IGLGSWVDTL

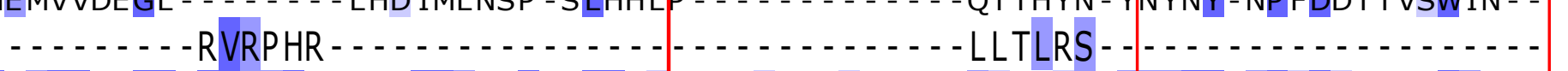

DGLLAGVGY - KVRSSE - LHQVAQN IE - RLENVI - - VNSSSD - - - - ISQ IAS - DTVHY - DPSD I - - GNWVDNL GLLANVGY - KVRSSE - LHQVAQNLE - RLESA I - VNSSSD - - - - ISQFAS - DTVHY - DPSD I - GNWVDNL GLLADVGY - KVKSSE - LHQVAQN IE - RLENVI - - VNSSD - - - - - ISQFAS - DT IHY - DPSD I - - GNWVDNL DGLLADVGY - KVKSSE - LHQVAQN IE - RLENV I - - VNSSSSD - - - ISQFAS - DTVHY - DPSD I - - GNWVDNL DELLAVVGY -KVRSSD - MAE VAQK IE - QLEEAM - - TNVETN - - - - ISSLSS - NTVHY - NPSD I - -STWLESM DELLAVVGY -KVRSSD - MAE VAQK IE - QLEE AM - - TNFDTG - . - - ISSLSS - DT IHF - NPSD I - -STWLESM DELLAVVGY -KVRSSD - MAE VAQKLE - RLEE AM - -GNVQDD - . - - LTDLSN - DAVHY - NPSD I - -SNWLQTM DELLAVVGY -KVRSSD - MAE VAQKLE - RLEE AM - -GNVQDD - - - - LPE ISN - DVVHY - NPSD I - -SNWLETM DELLAVVGY - KVRSSD - MAEVAQKLE - RLEEAM - -GN IQDG - - - - LKE LSD - GAAHY - NPSD I - -SNWLETM DELLAVVGY - KVRSSD - MAEVAQKLE - RLEEAM - -GNVQDG - - - - LKE LSD - DAVHY - NPSD I - -SNWLQTM DE LLAVVGY -KVRSSD - MAE VAQKLE - QLEE AM - - - - SDDT I - - - AHHLSS - DT IHF - NPAD I - - GTWLETM DELLAVVGY -KVKSSD - MAE VAQKLE - QLEHAM - GNFODODE A I IAQQLSN - DTVHY - NPSD I - SNWLKTM ELLAVVGY - KVKSSD - MAE VAQKLE - QLEQAMMGNNFHDHDEST I AQHLSN - DTVHY - NPSD I - SNWLQTM ELLAVVGY - KVKSSD - MAE VAQKLE - QLEQAM - -GNFQDQDEST IAQQLSN - DTVHY - NPSD I - SNWLQTM DELLAVVGY - KVKSSD-MAEVAQKLE - QLEQAMNGGNFQDQDEST I AQQLSN - DTVHY - NPSD I - - SNWLQTM DELLAA IGYKKLRSKDNMADVGQK LDQQLEAVMMDNNVNAREDGTSSNVASDDAVVHYDDPNDV - - FPWEKTM DELLEVLGY -KVRSSD - MADVAHKLE - KLEDVI - - VTTQQNG - - - ISNLATHHTVHY - NPTDL - - HGWVHNM DKLLAGVGY -KVCSSD - MADVAOKLE - OLEMVM - . - - DEDG - . - IPHLP T - DTVHY - DPTDL - - YGWVQN I DRLLAGVGY -KVCSSN - MVDVAHKLD - QLEMVM - . - - - EGDG - . - MSHLAN - NTVHY - DPTDL - - YGWVQN I DKLLAGVGY -KVCSSD - MVDVAHKLD-QLEMVM - . . - -EEDG - . - IPNLAS - DTVHY - DPTDL - - YGWVQN I DKLLAGVGY-KVCSSD-MVDVAHKLD - QLEMVM - - - - -EEDG - - - ISHLAS - NTVHY - DPTDL - - YGWVQN I

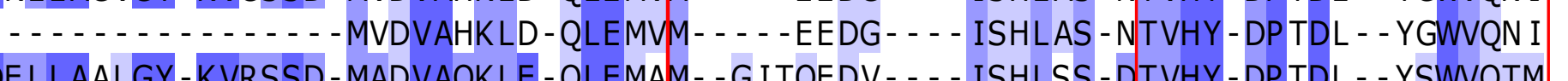

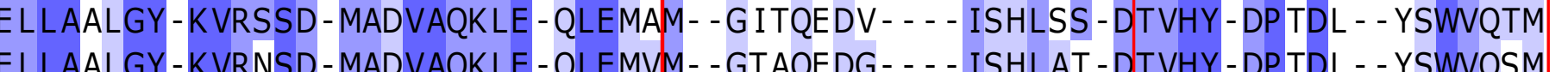
- LLALGY - KVRNSD - MADVAQKLE - QLEMVM - -GTAQEDG - . - ISHLAT DELLAALGY - KVRSSD - MADVAQKME - QLEMVM - - G IAQEDG - - - ISHLAS - DTVHY - DP TDL - - YSWVQTI

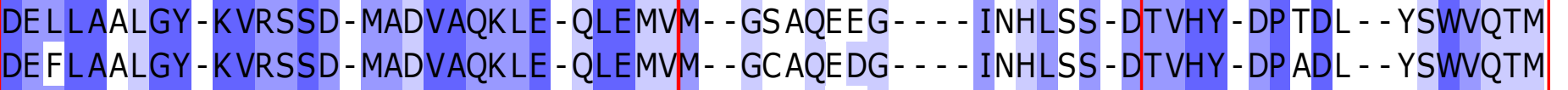
ELLAALGY - KVRSSD - MADVAQKLE - QLEMVM - -GS AQEEG - - - INHLSS - DTVHY - DPTDL - - YSWVQTM DELLAALGY - KVRSSD - MADVAQKLE - QLEMVM - -GS AQEEG - - - INHLSS - DTVHY - DPTDL - - YSWVQTM DE LLAALGY - KVRSSD - MADVAQKLE - QLEMVM - -GSAQEEG - - - ISHLAS - DTVHY - DPTDL - - YSWVQTM DE LLAALGY - KVRASD - MADVAQKLE - QLEMVM - - GCAQEDG - - - ISHLAS - DTVHY - DPTDL - - YSWVQSM DE LLAALGY - KVRASD - MADVAQKLE - QLEMVM - -GCAQEEG - - - ISHLAS - DTVHY - DPTDL - - YSWVQTM DELLAALGY - KVRASD - MADVAQKLE - QLEMVM - -GSAQEEG - - - ISHLSS - YTVHY - DPTDL - - HSWVQSM DELLAALGY - RVRASD - MADVAQKLE - QLEMVM - -GSAQEDG - - - ISHLAS - DTVHY - DPTDL - - HSWVQSM DELLAALGY - KVRASD - MADVAQKLE - QLEMVM - -GSAQEEG - - - ISHLAS - DTVHY - DPTDL - - HSWVQSM DELLAALGY -KVRASD-MADVAQKLE - QLEMVM - -GS AQEEG - - - ISHLAS -DTVHY-DPTDL - - HSW - - - 